\title{
Special Stains in Study of Infertile Endometrium
}

\author{
Mohasin Jakirhusain Halgale ${ }^{1 *}$, Meenal Vitthal Jadhav² and Nalini V. Kadgi ${ }^{2}$ \\ ${ }^{1}$ Department of Pathology, R.C.S.M. Government Medical College, Kolhapur, India \\ ${ }^{2}$ Department of Pathology, B.J. Medical College, Pune, India
}

\begin{abstract}
Background: Integrity of ovarian function is a prerequisite for successful implantation of ovum. The subtle changes in ovarian function can be detected by studying endometrial carbohydrates with special stains.

Material and Methods: 87 infertile and 30 normal endometria were studied with the help of Hematoxylin\& Eosin ( H\& E) stain and special stains like Best Carmine, Alcian blue PAS (with and without diastase digestion) and reticulin stain.

Results: Special stains were useful in emphasizing the cyclical activity of carbohydrates and stroma in normal endometrium. In infertile endometrium, they helped in precise classification and detection of corpus luteal deficiency, at times (8.7\% cases) missed on routine H\& E stain . Intrinsic abnormalities like polyps and tuberculous granulomas could be detected with ease on reticulin stain. On application of special stains, incidence of corpus luteal deficiency was $27.5 \%$, and that of anovulatory infertility and normal secretory endometrium was $34.5 \%$ each.
\end{abstract}

Conclusion: The special stains helped in detecting precise endometrial response and added to the information obtained by routine H\&E stain.

\section{Keywords: Endometrium, Infertility, Special Stains.}

\section{Introduction}

Endometrial carbohydrates namely glycogen and mucin show remarkable cyclical changes and are under hormonal control. These carbohydrates and integrity of their metabolism is of great importance in successful implantation. By detecting these substances, subtle changes in ovarian function can be detected. The simplest way of achieving this is by using special stains. ${ }^{[1]}$ The present study is attempted to highlight this aspect.

\section{Materials and Methods}

In this, case control study, prospectively registered 87 infertile women were investigated clinically and with pertinent investigations. Endometrial specimens were collected in the immediate premenstrual period by dilatation and curettage. 30 control endometrial specimens from fertile females of comparable age group being investigated or treated for non-hormonal causes acted as control.

The specimens were fixed and processed routinely and the slides were stained by $\mathrm{H} \& \mathrm{E}$ stain. The special stains employed were Alcian Blue PAS, Alcian Blue PAS with diastase digestion, Best carmine and Reticulin stain.The form of endometrial carbohydrate, its site and amount was noted and impression was offered as very low, low, moderate and high. The endometrial reticulin was noted for its thickness, frequency and form.

\section{Result}

Of the special the stains used for detection of carbohydrates, Best carmine gave unsatisfactory and erratic results and were never reproducible, but when successful the staining of glycogen was very crisp. Alcian blue PAS provided beautiful color contrast and helped in differentiating various components of carbohydrates. It stained acid mucin blue, imparted violet hue to mixture of mucins and magenta color to glycogen and neutral mucin (Figure 1). Detection of glycogen depended on its disappearance on diastase digestion. This was rather inconvenient to interpret and quantify. The detection of endometrial carbohydrate as a single entity (on Alcian blue PAS without diastase digestion) was more convenient than detecting glycogen and mucin separately. The Reticulin stain helped in detecting very small areas of pseudodecidual change, thus making it easy to date the endometrium.

The endometrial mucin and glycogen were present in glands and stroma, both intracellularly and extracellulerly. The glycogen was present as fine or coarse granules in glandular epithelium, lumen and in stroma. The mucin was present mainly in glandular lumina, apical borders 
of glandular epithelium and in the stroma in the form of large and small masses and granules respectively. The mucin could not be appreciated satisfactorily in cytoplasm of glandular epithelium. The distribution of carbohydrates varied in a same specimen from place to place and from specimen to specimen of a similar endometrial date, though the overall endometrial carbohydrate content was similar. It was more convenient to offer general impression of total carbohydrate content on a slide than noting it differently.

In normal endometrium carbohydrates and reticulin exhibited cyclical activity. The carbohydrates were absent in early proliferative phase, appeared in very low quantities in mid-proliferative phase and mildly increased but were still low in late proliferative phase. They were present in moderate amounts in early secretory phase and reached their peak in mid-secretory phase (Figure 2). They were present in moderate quantities in late secretory phase, where they were accumulated mainly in glandular lumina and in the stroma.

The reticulin formed thin periglandular baskets and was present as thin discrete fibers in interglandular space in proliferative phase. In secretory phase, periglandular baskets became thick, perivascular network appeared and became progressively thick. Interglandular fibers formed incomplete mosaics around pseudodecidual cells in late secretory phase (Figure 3 ).

Classification of endometrial changes observed on $\mathrm{H} \& \mathrm{E}$ stain in infertility was as shown in Table 1.

Application of special stains did not reveal any additional abnormalities in cases with proliferative phase. The findings on endometrial carbohydrates detected in secretory endometrium are summarized in Table 2.

Close look at Table 2 shows that on application of special stains, 10 additional cases with low carbohydrate content could be diagnosed as corpus luteal deficiency and six cases with corpus luteal deficiency previously classified as coordinate delay with poorly developed glands in terms of carbohydrate content can be reclassified as dissociate delay. This implies the fact that there were 22 cases of dissociate delay and two cases of coordinate delay after application of special stains.

The cases of tuberculous endometritis showed rings of reticulin around granulomas, making it easy to detect them (Figure 4). The endometrial polyp showed thick interglandular fibers and very thick perivascular network.

Table 1: Morphological changes in infertile endometrium on H. \& E. Stain.

\begin{tabular}{|l|c|c|}
\hline Endometrial change & No of cases n= 87 & Percentage \\
\hline A. Proliferative phase & 30 ) & 13 \\
\hline Deficient proliferation ( Early proliferative change ) & 14 \\
\hline $\begin{array}{l}\text { Intermediate proliferation ( Late proliferative change with } \\
\text { occasional cystically dilated gland ) }\end{array}$ & 3 \\
\hline Irregular proliferation (stromal \& glandular hyperplasia) & $(54)$ \\
\hline B. Secretory phase & 40 \\
\hline Adequate secretory response (late secretory change) & 8 \\
\hline $\begin{array}{l}\text { Corpus luteal deficiency with Co-ordinate apparent delay } \\
\text { (normal early or mid secretory change) }\end{array}$ & 6 \\
\hline $\begin{array}{l}\text { Corpus luteal deficiency with Dissociate delay (early or mid secretory change } \\
\text { with discordant glandular \& stromal development) }\end{array}$ & 62 \\
\hline C. Intrinsic abnormality & $(3)$ \\
\hline Tuberculous endometritis & 2 \\
\hline Endometrial polyp & 1 \\
\hline
\end{tabular}

Table 2: Endometrial carbohydrates in secretory phase.

\begin{tabular}{|l|c|c|c|}
\hline \multirow{2}{*}{ Endometrial change (H\&E) (n) \% } & \multicolumn{3}{|c|}{ Status of carbohydrates, (n) \% } \\
\cline { 2 - 4 } & Adequate glycogen \& mucin & Low glycogen \& mucin & Low mucin \\
\hline Adequate secretory response (40) 45.9 & $(30) 34.5$ & (8) 9.2 & $(2) 2.29$ \\
\hline $\begin{array}{l}\text { Corpus luteal deficiency with Co-ordinate } \\
\text { apparent delay (8) } 9.2\end{array}$ & $(2) 2.29$ & (4) 4.6 & $(2) 2.29$ \\
\hline $\begin{array}{l}\text { Corpus luteal deficiency with Dissociate } \\
\text { delay (6) } 6.8\end{array}$ & - & (6) 7 & - \\
\hline Total (54) 61.9 & (32) 36.8 & (18) $\mathbf{2 0 . 6}$ & (4) 4.5 \\
\hline
\end{tabular}




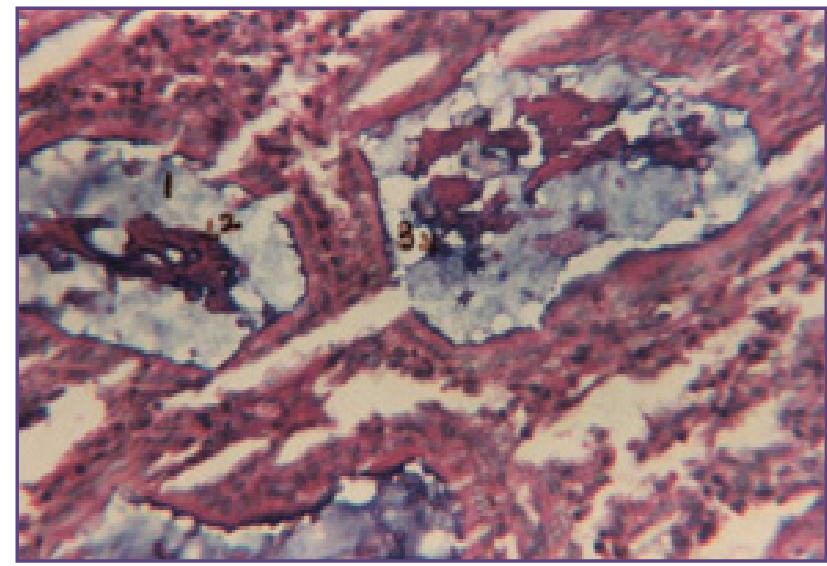

Fig. 1: Alcian blue PAS, X400, normal secretory endometrium, lumina showing blue Acid mucin\& magenta Neutral mucin and Glycogen.

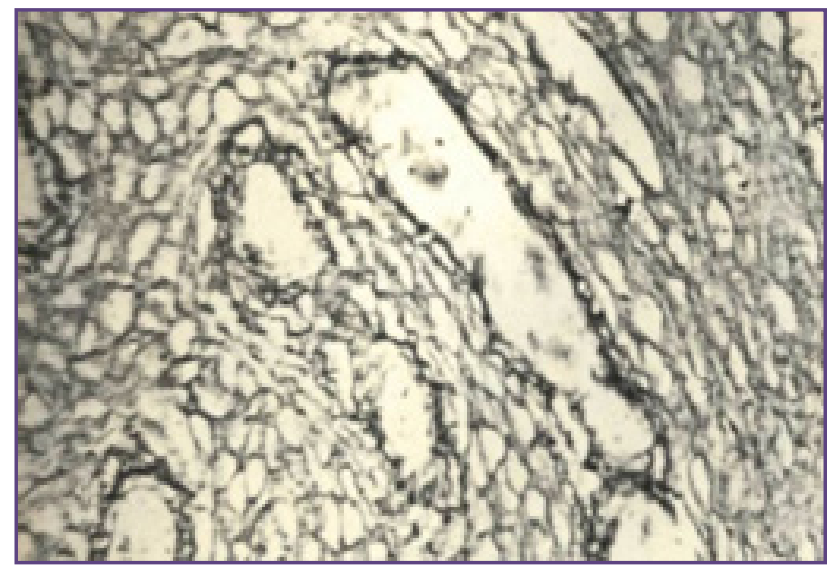

Fig. 3: Reticulin stain, $X$ 400, normal endometrium with late secretory phase with thick baskets of reticulin around glands and mosaic of reticuline around pesudodecidual cells.

\section{Discussion}

Study of endometrial histology provides useful information about its hormonal preparation and its role in implantation. Application of special stains, particularly those designed to study glycogen and mucin makes this study more meaningful. By this method it is possible to note the subtle changes in hormonal balance. These in turn help in precise classification of endometrial changes associated with infertility which need to be treated differently. ${ }^{[1]}$ In present study the interesting observations of reclassification of cases in secretory phase on application of special stains emphasize this role (Table 2).

The unovulatory endometrium calls for ovulation inducing treatment. To be precise, deficient proliferation can be treated with estrogen priming with clomiphene and progesterone. Intermediate and irregular proliferation is treated with clomiphene. The infertile endometrium

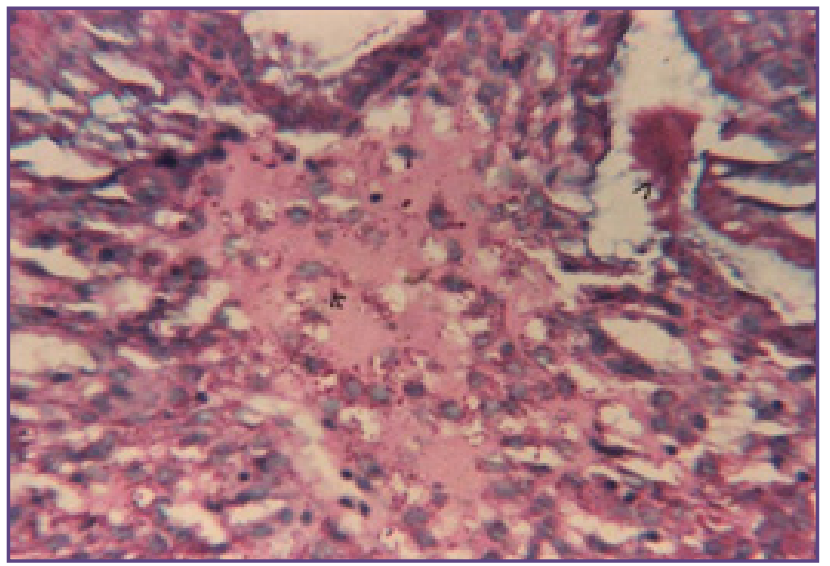

Fig. 2: Alcian blue PAS, X400, normal midsecretory endometrium, with abundant carbohydrate masses in lumen and granules in edematousstroma.

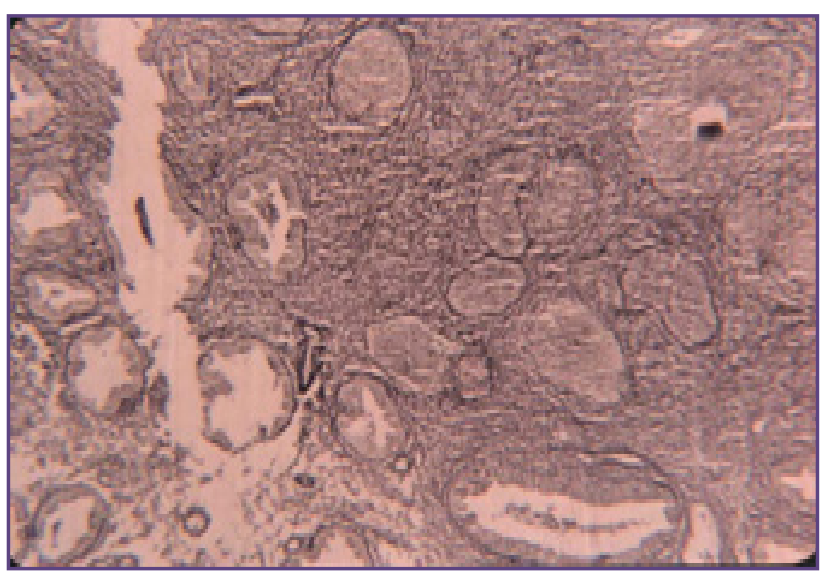

Fig. 4: Reticulin stain, X 400, Tuberculous endometrium, rings of reticulinarroundtuberculous granulomas

with secretory change can be a result of deficiency of progesterone which can be absolute or relative. This results in delaying of endometrial maturation. With absolute progesterone deficiency, there occurs asynchronous delayed maturation of glands and stroma which is labeled as Corpus luteal deficiency with Dissociate delay which needs to be treated with FSH in first half of cycle followed by hCG and progesterone. On the other hand when normal progesterone secretion is counteracted by hyperestrogenism coming from persistent graffian follicle the maturation of glands and stroma is delayed but coordinated and apparent. ${ }^{[1,2]}$ In such cases treatment with tamoxifen which is a partial antagonist of estrogen, in proliferative phase might help in lengthening the secretoty (luteal) phase and improving its glycogen content. ${ }^{[3]}$

It will be worthwhile to discuss the classification of endometrial changes undertaken in present study. ${ }^{[1]} \mathrm{On} \mathrm{H}$ 
\& E stain endometrium classified as deficient proliferation showed retarded glandular and stromal development with the glands resembling those of early or mid-proliferative endometrium. Endometrium placed in the group of intermediate proliferation showed glands comparable to those in late proliferative phase with occasionally cystically dilated glands. Endometrium classified as irregular proliferation showed glandular and stromal hyperplasia. Endometrium showing adequate secretory response was in late secretory phase as expected in view of the immediate premenstrual sampling. Eight cases which were in early or mid-secretory phase with co-ordinate glandular and stromal development were classified as corpus luteal deficiency with co-ordinate apparent delay. Six cases showed poorly convoluted glands with a variation in development of glands and stroma, from region to region and dissociation of development between glands and stroma. These were labeled as corpus luteal deficiency with dissociate delay. Together these 14 cases were classified as corpus luteal deficiency on $\mathrm{H} \& \mathrm{E}$ stain.

On reclassification with special stains the frequency of corpus luteal deficiency in secretory endometrium rose from $16(18.3 \%)$ to $22(25.1 \%)$ cases [Table $1 \& 2$ ]. In literature, the frequency of corpus luteal deficiency in infertile secretory endometrium varies from $7.5 \%$ to $20 \%{ }^{[4,5]}$

In present study $40.7 \%$ cases of ovulatory infertility show deficiency of endometrial carbohydrates. The experience is shared by others. ${ }^{[4 .}{ }^{5]}$ Maeyama et.al have shown that this deficiency can be corrected at least in vitro by addition of progestero ne. ${ }^{[6]}$ It is quite interesting to note that in the present series, deficiency of mucin occurred along with deficiency of glycogen in some cases of ovulatory infertility and few additional cases showed deficiency of mucin alone. In view of these findings, study of endometrial carbohydrate as a single entity is advocated rather than studying them differently. This also obviates need for supplementation of Alcian blue stain to PAS stain. Use of PAS stain, which belongs to routine histology laboratory setup, can be thus advocated in routine protocol of endometrial evaluation for infertility.

In literature authors like Schoon et al have attempted special stains and immunohistochemistry to confirm the endometrial maldifferentiation of infertile endometrium and advocated its inclusion in routine evaluation of infertile endometrium. ${ }^{[7]}$ Use of special stains in knowing the glandular secretion pattern in abnormal endometrium is practiced by some other authors as well. ${ }^{[8,9,10]}$

\section{Conclusion}

Special stains seem to open up multiple avenues of evaluation of infertile endometrium which will go long way in treating this condition.

\section{Acknowledgements}

Dr. Sharmila Shinde and staff of Department of Pathology, B. J. Medical College, Pune for their valuable contribution.

\section{References}

1. Dallenbach G-Hellweg.Histopathology of the endometrium. 4thed. Berlin, Heidelberg, New York, London, Paris, Tokyo: Springer-Verlag; 1987.

2. Dallenbach- Hellweg G. The endometrium of infertility. A review. Pathol Res Pract. 1984 ; 178(6):527-37.

3. Spirtos NJ, Yurewicz EC, Moghissi KS, Magyar DM, Sundareson AS, Bottoms SF. Pseudocorpus luteum insufficiency: A study of cytosol progesterone receptors in human endometrium. Obstet Gynecol 1985; 65: 535-40.

4. Zawar MP, Deshpande NM, Gadgil PA, Mahanta AA. Histopathological study of endometrium in infertiity. Indian J Pathol Microbiol. 2003; 46: 630-3.

5. Sahasrabudhe NS, Shinde S, Jadhav MV. Endometrium in infertility. Indian Journal of Obstetrics and Gynaecology. 2001; 51: 100-2.

6. Meayama M, Ikuzo S, Matsuol I, Nakahara K. Glycogen estimation by rapid enzymatic method in human endometrium. Glycogen content in the endometrium of infertile patients during the menstrual cycle. Fertil Steril.1977; 28: 159- 2 .

7. Schoon HA, Wiegandt I, Schoon D, Aupperle H, Bartmann $\mathrm{CP}$. Functional disturbances in the endometrium of barren mares: a histological and immunohitological study. J Reprod Fertil Suppl. 2000; 56: 381-91.

8. Hoffmann C,Bazer FW, Klug J, Aupperle H, Ellenberger $\mathrm{C}$, Schoon HA. Immunohistochemical and histochemical identification of proteins and carbohydrates in the equine endometrium Expression patterns for mares suffering from endometrosis. Theriogenology. 2009;71(2):264-74.

9. Devi LS, Singh MR, Singh LR, Debnath K. The histological and histochemical study of endometrium in dysfunctional uterine bleeding. J Med Soc. 2012; 26: 167-70.

10. Salma M, Kouser T, Nasar MA. Endometrial Hyperplasia: a 5 - Years Retrospective Study. IJPO. 2016; 3(2): 221-25.

*Corresponding author:

Mohasin Jakirhusain Halgale, 816/3, Plot no. 6, Karande Mala, Devane Colony, Opp. Sriram Residency, Kolhapur - 416003

Phone: +91 9923431297

Email: mohsinhalgale@yahoo.in

Financial or other Competing Interests: None. 